\title{
Effect of Cyanophycin Metabolism in Recombinant Sinorhizobium (Ensifer) meliloti 1021 on the Symbiosis with Alfalfa (Medicago sativa)
}

Yasser Abd-El-Karem ${ }^{1}$, Rudolf Reichelt ${ }^{2}$, Martin Krehenbrink ${ }^{3}$ and Alexander Steinbüchel ${ }^{1,4 *}$

${ }^{1}$ Institut für Molekulare Mikrobiologie und Biotechnologie, Westfälische Wilhelms-Universität, Münster, Germany

${ }^{2}$ Institut für Medizinische Physik und Biophysik, Westfälische Wilhelms-Universität, Münster, Germany

${ }^{3} \mathrm{Cysal} \mathrm{GmbH}$, Münster, Germany

${ }^{4}$ Environmental Sciences Department, King Abdulaziz University, Jeddah, Saudi Arabia

\begin{abstract}
The aim of this study was to investigate the effect of the synthesis and degradation of the nitrogen-rich polymer cyanophycin in rhizobia on symbiotic nitrogen fixation and crop yield in legumes. For this, cyanophycin synthetase from Anabaena sp. PCC7120 was expressed in the bacteroids of the symbiont S. meliloti 1021 either alone or together with an intracellular cyanophycinase from the same bacterium, either in the wild type or in a polyhydroxybutyratenegative $\left(\mathrm{PHB}^{-}\right)$mutant, and the effect on the growth of alfalfa host plants was studied. All strains induced the formation of nitrogen-fixing nodules in the host, but clear differences in various parameters were noticeable. Alfalfa plants infected with the wild type expressing only cyanophycin synthetase showed significantly lower shoot nitrogen contents and higher nitrogen fixation rates than plants inoculated with wild type, but the wild type phenotype was exceeded in S. meliloti 1021 expressing cyanophycinase in addition to cyanophycin synthetase. Growth of plants infected with the PHB- mutant expressing only cyanophycin synthetase was severely impaired in comparison to growth of plants infected with the PHB- mutant expressing both or neither of the two proteins. Transmission electron micrographs of sections of nodules induced by wild type S. meliloti 1021 producing cyanophycin synthetase and cyanophycinase showed that the rough endoplasmic reticulum and Golgi membranes were extended further in comparison to the nodules induced by the wild type, the PHB mutant, or all other recombinant $S$. meliloti strains, indicating higher metabolic activities in these nodules.
\end{abstract}

Keywords: Cyanophycin; Nitrogen fixation; Rhizobia; Symbiosis; Crop yield; Alfalfa

\section{Abbreviations \\ ATP: Adenosine Triphosphate; PCR: Polymerase Chain Reaction}

\section{Introduction}

Cyanophycin (multi-L-arginyl-poly-L-aspartic acid, cyanophycin granule polypeptide or CGP) was discovered over one hundred years ago during light microscopic examinations of cyanobacteria [1]. The polymer consists of equimolar amounts of arginine and aspartic acid arranged as a polyaspartate backbone with arginine moieties linked to the $\beta$-carboxyl group of each aspartate via a peptide bond [2]. CGP is synthesized in the cytoplasm of most cyanobacteria [3-5] and also of several non-photosynthetic bacteria [6,7] and deposited in the cells as water-insoluble membrane-less granules.

CGP is usually insoluble in water at physiological $\mathrm{pH}$, but is soluble under acidic $(\mathrm{pH}<2)$ or alkaline $(\mathrm{pH}>9)$ conditions [8]. In addition, a water-soluble form of CGP was observed in cells of a recombinant Escherichia coli strain [7]. Intracellular synthesis of CGP is catalyzed by cyanophycin synthetase, which is encoded by $c p h A$ [7]. Degradation of CGP is catalyzed by two different types of enzymes acting consecutively: (i) an exopeptidase also referred to as cyanophycinase (CphB), produces $\beta$-Asp-Arg dipeptides [9], and (ii) a peptidase that hydrolyzes these dipeptides to the amino acid monomers [10]. A detailed overview of the distribution of CGP metabolism genes and thorough in silico analyses of the respectives genes and of the gene products has been published [11]. The genome of Anabaena sp. PCC7120 harbors two cph clusters, each consisting of $c p h B$ and $c p h A$. However, it was shown that the cyanophycin synthetase of the first cluster (CphA1) contributed more to the synthesis and accumulation of CGP than the second cyanophycin synthetase (CphA2) [12].

The cyanophycin synthetase genes $(c p h A)$ of many cyanobacteria
[13-15] as well as those of the chemotrophic bacteria Acinetobacter sp. strain DSM 587 [6] and Desulfitobacterium hafniense DCB-2 [7] were cloned and expressed in E. coli. Heterologous expression of $c p h A$ was also demonstrated in recombinant strains of Ralstonia eutropha, Corynebacterium glutamicum, Pseudomonas putida and Bacillus megaterium [16]. CGP has also been produced in a few eukaryotes [17-21].

The Gram-negative soil bacterium Sinorhizobium meliloti fixes atmospheric nitrogen in symbiotic association with its host plants, including Medicago truncatula [barrel medic] and Medicago sativa (alfalfa) [22]. A successful symbiosis involves a series of specific interactions between the legume host and the rhizobial symbiont [22]. Symbiosis is initiated by the plant root by the release of flavonoids, which attract $S$. meliloti cells from the surrounding environment and induce the formation of nodulation (Nod) factors by the bacteria. The Nod factors then induce root hair curling and root nodule development. S. meliloti cells invade the developing root nodules via infection threads, extended invaginations of the root hair cell membrane [23]. From these, bacteria are then released into the cells of the developing nodule [24]. Once released from the infection threads, the bacterial cells differentiate into bacteroids that are able to convert atmospheric

\footnotetext{
*Corresponding author: Alexander Steinbüchel, Institut für Molekulare Mikrobiologie und Biotechnologie, Westfälische Wilhelms-Universität, Münster, Germany, Tel: +49-251-8339821; Fax: +49-251-8338388; E-mail: steinbu@uni-muenster.de
}

Received July 20, 2016; Accepted August 01, 2016; Published August 08, 2016

Citation: Abd-El-Karem Y, Reichelt R, Krehenbrink M, Steinbüchel A (2016) Effect of Cyanophycin Metabolism in Recombinant Sinorhizobium (Ensifer) meliloti 1021 on the Symbiosis with Alfalfa (Medicago sativa). J Microb Biochem Technol 8: 375381. doi: 10.4172/1948-5948.1000312

Copyright: (c) 2016 Abd-El-Karem Y, et al. This is an open-access article distributed under the terms of the Creative Commons Attribution License, which permits unrestricted use, distribution, and reproduction in any medium, provided the original author and source are credited. 
nitrogen to ammonia [25]. The host plant supplies bacteroids with carbon compounds, including dicarboxylic and amino acids [26], which are used by the bacteroids to support the reduction of nitrogen to ammonia in amounts sufficient for plant growth [24].

The cyanophycin synthetase and cyanophycinase genes (cphA1 and cphB1) from Anabaena sp. PCC7120 were heterologously expressed in wild type $S$. meliloti 1021 and in a phbC-negative mutant of this strain. CGP was accumulated up to $44 \%$ (wt/wt) of cell dry weight in the recombinant cells harbouring only $c p h A 1$ alone without $c p h B 1$. In contrast, no CGP accumulation was observed in cells harbouring both $c p h A 1$ and $c p h B 1$. The aspartate-arginine dipeptides released from CGP degradation were shown to be used by the cells to fuel PHB biosynthesis [27].

In rhizobia inhabiting indeterminate nodules, such as $S$. meliloti, M. ciceri, and R. leguminosarum bv. viciae, PHB accumulates in freeliving cells, but generally not in bacteroids [28]. The reason for this is unknown, but GC analysis of bacteroids of M. ciceri CC1192 has shown that small amounts of PHB are accumulated [29]. PHB granules were also observed in mature bacteroids of R. leguminosarum bv. viciae carrying mutations in the broad-specificity amino acids transporters aap and bra [26]. Blocking the amino acid cycling pathway between the plant and the bacteroid prevents the synthesis of aspartate from oxaloacetate, which increases carbon flow from dicarboxylate to pyruvate, which in turn is channelled into PHB synthesis [26].

This study was aimed at investigating the effect of CGP biosynthesis alone or its biosynthesis and degradation in the nodules induced by recombinant cells of strains $S$. meliloti 1021 (pBBR1MCS-3::cphA $1_{7120}$ ), S. meliloti 1021 phbCSKm (pBBR1MCS-3::cphA1 $1_{7120}$ ), S. meliloti 1021 (pBBR1MCS-3::cphB1 ${ }_{7120}:: c p h A 1_{7120}$ ), and S. meliloti 1021 phbC $\Omega \mathrm{Km}$ (pBBR1MCS-3::cphB1 $1_{7120}:: c p h A 1_{7120}$ ) on the symbiosis with alfalfa plants.

\section{Materials and Methods}

\section{Bacterial strains and cultivation conditions}

The bacterial strains and plasmids used in this study are listed in Table 1. Cells of S. meliloti were grown at $30^{\circ} \mathrm{C}$ in Trypton Yeast (TY) medium [30]. Antibiotics were used at the following concentrations: kanamycin $(300 \mathrm{mg} / \mathrm{l})$, streptomycin $(100 \mathrm{mg} / \mathrm{l})$, and tetracycline $(12.5 \mathrm{mg} / \mathrm{l})$. Aspartic acid and arginine were added as supplements at concentrations of $0.2 \%$ (wt/vol). For induction $0.1 \mathrm{mM}$ isopropyl- $\beta-\mathrm{D}$ thiogalactopyranoside (IPTG) was added.

\section{Nodulation experiments}

Seeds of alfalfa (Medicago sativa) var. "Eugenia" (Revierberatung Wolmersdorf, Germany) were surface-sterilized by soaking in $70 \%$ ethanol for $1 \mathrm{~min}$, followed by $10 \mathrm{~min}$ soaking in $1 \%$ (vol/vol) freshly prepared solution of sodium hypochlorite and rinsed several times with sterile $\mathrm{H}_{2} \mathrm{O}$ [31]. The treated seeds were germinated on $1 \%$ water agar in sealed Petri plates at room temperature in the dark for $48 \mathrm{~h}$.
Germinated seedlings were transferred to plant growth tubes (50 $\mathrm{ml}$ polypropylene falcon centrifuge tube, Greiner Bio-One $\mathrm{GmbH}$, Germany) containing $25 \mathrm{ml}$ sterile nitrogen-free $\mathrm{B}+\mathrm{D}$ plant nutrient solution solidified using $1 \%$ agar [32] covered with $0.5-1 \mathrm{~cm}$ of sterile, washed vermiculite saturated with $\mathrm{B}+\mathrm{D}$ plant nutrient solution at one seedling per tube. The outer surface of plant growth tubes were covered with aluminium foil surrounding only the medium area to provide dark conditions for the plant root. Four days after germination, seedlings were inoculated with the $S$. meliloti strains. The cells were grown for $48 \mathrm{~h}$ in TY medium [supplemented with the appropriate antibiotics, $0.1 \mathrm{mM}$ IPTG, $0.2 \%$, wt/vol arginine, and $0.2 \%$, wt $/ \mathrm{vol}$ aspartic acid, harvested by centrifugation $(15 \mathrm{~min}, 2,800 \mathrm{x} g$ ), washed twice with saline solution $(0.9 \%$, wt $/ \mathrm{vol} \mathrm{NaCl})$, and diluted to an optical density at $600 \mathrm{~nm}$ of 0.2 . Each germinated seedling was inoculated with 0.5 $\mathrm{ml}$ of the bacterial suspension, and each of the control seedlings was mock inoculated with $0.5 \mathrm{ml}$ of saline solution $(0.9 \%$, wt $/ \mathrm{vol} \mathrm{NaCl})$. After inoculation, the tubes were wrapped with semi-transparent parafilm (Rio Tinto Alcan, Canada) until the growing plants reached the top of the tube; it was removed afterwards. Each treatment included 48 replicates, where each replicate consisted of one tube containing one plant. The plants were placed in randomized blocks in a growth chamber $\left(16 \mathrm{~h}\right.$ at $25^{\circ} \mathrm{C}$ \{day\}, $8 \mathrm{~h}$ at $20^{\circ} \mathrm{C}$ \{night $\}$, light intensity 18,000 lux and $60 \%$ relative humidity). The inoculated plants were grown for 55 days and watered with nitrogen-free $\mathrm{B}+\mathrm{D}$ plant nutrient solution as necessary.

Nitrogenase activity in nodulated roots [30], shoot length, root length, number of leaves, number of nodules, nodule fresh weight, shoot dry weight and $\% \mathrm{~N}(\mathrm{wt} / \mathrm{wt})$ of shoot dry weight [33] were determined after the plants had been harvested. Bacteria were isolated from the nodules at 35 and 55 days. The nodules were surface-sterilized by soaking for five to ten seconds in $95 \%$ ethanol, followed by two to four minutes soaking in freshly-prepared $3 \%$ ( $\mathrm{vol} / \mathrm{vol}$ ) solution of sodium hypochlorite, rinsed several times with sterile $\mathrm{H}_{2} \mathrm{O}$, then crushed in a few drops of TY medium. The resulting suspension was streaked onto TY agar plates supplemented with the appropriate antibiotics.

\section{Nitrogenase activity assay}

Nitrogen fixation activity was determined by the acetylene reduction method as follows: Seven millilitres of acetylene gas were injected into stoppered $70-\mathrm{ml}$ bottles containing alfalfa root segments with nodules from 35- or 55-day old plants inoculated with S. meliloti stains. The bottles were incubated for 1-3 $\mathrm{h}$ before analysis at room temperature. A $100 \mu \mathrm{l}$ gas sample from each tube was analyzed for the presence of acetylene and ethylene using an Agilent 6850 gas chromatograph (Waldbronn, Germany) equipped with a BP21 capillary column $[0.25 \mathrm{~mm}$ diameter, $250 \mathrm{~nm}$ film thickness, $50 \mathrm{~m}$ length, SGE Darmstadt, Germany] and a flame ionization detector. The flow rate of the hydrogen carrier gas was set at $30 \mathrm{ml} / \mathrm{min}$. The injection temperature was $100^{\circ} \mathrm{C}$, and the column temperature was $35^{\circ} \mathrm{C}$. Under these conditions, ethylene typically eluted after $4.405 \mathrm{~min}$, while acetylene eluted after $4.530 \mathrm{~min}$.

\begin{tabular}{|c|c|c|}
\hline Strains or plasmids & Relevant characteristics & References or sources \\
\hline $\begin{array}{r}\text { S. meliloti } \\
\text { Rm1021 } \\
\text { Rm4775 }\end{array}$ & $\begin{array}{l}\text { SU47 str-21, Smr } \\
\text { S. meliloti } 1021:: p h b C \Omega \mathrm{Km}^{r}\end{array}$ & $\begin{array}{l}{[47]} \\
{[27]}\end{array}$ \\
\hline \multicolumn{3}{|l|}{ Plasmids } \\
\hline pBBR1MCS-3::cphA1 ${ }_{7120}$ & $\begin{array}{l}\text { Tc', mob+, tra, lacPOZ', cphA1 in addition to carboxy-terminal region of } c p h B 1 \text { from Anabaena } \\
\text { sp. PCC7120 collinear to lacPOZ' }\end{array}$ & \\
\hline pBBR1MCS-3::cphB1 $1_{7120}:: c p h A 1_{7120}$ & pBBR1MCS-3 harbouring $c p h B 1$ and $c p h A 1$ from Anabaena sp. strain PCC7120 & [27] \\
\hline
\end{tabular}

Table 1: Bacterial strains and plasmids used in this study. Abbreviations: $\mathrm{Km}^{r}$, Kanamycin resistance; Smr , Streptomycin resistance; Tcr ${ }^{r}$ Tetracycline resistance. 


\section{DNA manipulation}

The following oligonucleotides were used as primers to amplify $c p h A 1_{7120}$ or the $c p h B 1_{7120}+c p h A 1_{7120}$ gene cluster by colony PCR from bacterial colonies recovered from surface-sterilized alfalfa nodules induced by the recombinant $S$. meliloti strains after 35 or 55 days post-inoculation: for amplifying $c p h A 1_{7120}$, primer P1 (5'-GCTATGGCGATCGCTTCCATCTGTACCAAAGG-3') and primer P2 (5'-GGTTTTACCTAGTGCGCTTCCCA-3') were used; for amplifying the cph1 gene cluster $(c p h B 1+c p h A 1)$, primer P3 (5'-TTTGGGGACACGGGACATACA-3') and primer $\mathrm{P} 2$ were used. The oligonucleotides were purchased from Thermo Scientific (Germany). For the amplification reaction Taq polymerase from Invitrogen [San Diego, CA] was used according to the instructions of the manufacturer.

\section{Transmission electron microscopy}

Nodules taken 35 and 55 days after inoculation with S. meliloti strains were fixed with $2.5 \%$ glutaraldehyde in $0.1 \mathrm{M}$ phosphatebuffered saline (PBS; pH 7.3) according to Sörensen [34] for $1 \mathrm{~h}$. After washing three times with PBS (30 min each), nodules were post-fixed in $1 \%$ osmium tetroxide in $0.1 \mathrm{M}$ PBS ( $\mathrm{pH} 7.3$ ) for $1.5 \mathrm{~h}$ and washed once with PBS (pH 7.3) for $10 \mathrm{~min}$. The water was then removed by using a graded water/ethanol series $(30,50,70,90$ and $96 \%$ ethanol followed by two changes of absolute ethanol) followed by propylene oxide (30 min each). To obtain thin sections, the dehydrated samples were embedded in Spurr's resin with propylene oxide [35] at the following mixture ratios (1:3 for $4 \mathrm{~h}, 1: 1$ for $4 \mathrm{~h}, 3: 1$ for $4 \mathrm{~h}$, respectively). The samples were soaked two times in Spurr's resin without propylene oxide for 4-8 $\mathrm{h}$ for the first soak and overnight for the second soak. The final embedding and polymerization was done in fresh Spurr's resin without propylene oxide overnight at $70^{\circ} \mathrm{C}$. After polymerization, longitudinal ultrathin sections with a thickness of 70-80 nm were cut with an ultramicrotome (Leica Mikroskopie und Systeme, Wetzlar, Germany) using a diamond knife (Diatome AG, Biel, Switzerland). The sections were then placed on 200-mesh copper grids that were covered with a thin film of pioloform. Sections were then stained for 30 min with saturated uranyl acetate in 50\% ethanol. After brief washing with double-distilled water, the sections were stained for five minutes with lead citrate according to Reynolds [36]. After brief washing with double-distilled water, imaging was performed with an $80 \mathrm{kV}$ electron microcope 'EM Philips 410' (Eindhoven, Netherlands).

\section{Statistical analysis}

All data for nitrogenase activity in nodulated roots as well as for shoot length, root length, number of leaves, number of nodules, nodule fresh weight, shoot dry weight, or $\% \mathrm{~N}$ (wt/wt) of shoot dry weight for plants 55 days after inoculation are given as means with standard errors. The statistical significance $\mathrm{P}<0.05$ for root length and number of nodules, $\mathrm{P}<0.01$ for nitrogenase activity, shoot length, number of leaves, nodule fresh weight, shoot dry weight, and \%N (wt/wt) of shoot dry weight of the results was assessed using Duncan's $t$ test.

\section{Results}

Alfalfa seedlings were inoculated with wild type S. meliloti 1021 (Rm1021), the phbC-negative mutant S. meliloti 1021 phbC $\Omega \mathrm{Km}$ (Rm4775), or with recombinant strains harbouring plasmids pBBR1MCS-3::cphA $1_{7120}$ or pBBR1MCS-3::cphB1 $1_{7120}:: c p h A 1_{7120}$. The inoculated plants were grown for 55 days in a growth chamber under controlled conditions as described in the Materials and Methods section. All strains induced elongate, cylindrical, pink, nitrogen-fixing nodules similar to the nodules induced by the wild type strain.
The results of time course analysis of plant growth, nodule development, and nitrogen fixation of alfalfa plants inoculated with the various $S$. meliloti strains are shown in Figures 1 and 2. Alfalfa plants inoculated with the $p h b C$-negative mutant $\mathrm{Rm} 4775$, which is unable to synthesize $\mathrm{PHB}$, demonstrated an insignificantly $[\mathrm{P}>0.01]$ higher acetylene reduction activity 55 days after inoculation in comparison to the acetylene reduction activity levels of plants inoculated with the wild type (Figure 2). However, they showed fewer nodules, significantly $[P<0.01]$ reduced shoot dry mass, and lower shoot nitrogen content (Figure 1 and Table 2). The plants inoculated with the recombinant phbC-negative mutant harbouring cphAl showed significantly $(P<0.01)$ lower shoot dry mass, shoot nitrogen content, acetylene reduction activity, and nodule fresh weight as well as a significantly $(P<0.05)$ lower number of nodules (Table 2$)$, and also much poorer growth (Figure 3 ) in comparison to alfalfa plants inoculated with the wild type strain. Furthermore, these plants showed the lowest growth of all inoculated plants in the experiment (Figures 1 and 2) (Table 2).

In contrast, plants inoculated with the recombinant phbCnegative mutant harbouring $c p h B 1$ in addition to $c p h A 1$ performed very differently. Higher acetylene reduction activity and nodule fresh weights were obtained with plants inoculated with this strain in comparison to plants inoculated with either the wild type strain or the $p h b C$-negative mutant or the recombinant $p h b C$-negative mutant harbouring only cphA1 (Figures 1 and 2). Moreover, the shoot dry weights of these plants were slightly higher than of those infected with the $p h b C$-negative mutant and significantly $(P<0.01)$ higher than of those infected with the recombinant $p h b C$-negative mutant harbouring only cphA1 (Table 2 ).

The root systems of the plants 55 days after inoculation with the wild type strain showed a significantly higher acetylene reduction activity $(32 \%)$ than plants inoculated with the recombinant strain Rm1021 harbouring both $c p h B 1$ and $c p h A 1$. In contrast, the latter plants exhibited enhanced growth as indicated by (albeit insignificantly, $\mathrm{P}>0.01$ ) higher nodule fresh weight, shoot dry weight, and shoot nitrogen content. The number of nodules was also (insignificantly, $\mathrm{P}>0.05$ ) higher (Table 2). Furthermore, the same plants showed an insignificantly $(\mathrm{P}>0.01)$ higher shoot dry weight, but a significantly $(P<0.01)$ higher shoot nitrogen content in comparison to the plants inoculated with the recombinant strain of $\mathrm{Rm} 1021$ harbouring only cphA1 (Table 2).

On the other hand, the plants inoculated with the recombinant Rm1021strain harbouring only cphA1 without $c p h B 1$ revealed approximately the same shoot dry weights, insignificantly higher number of nodules $(\mathrm{P}>0.05)$, higher nodule fresh weight $(\mathrm{P}>0.01)$, lower shoot nitrogen content $(P>0.01)$, and significantly $(P<0.01]$ higher acetylene reduction activity in comparison to plants infected with the wild type strain.

\section{Plasmid test assay}

A 3,105-bp fragment corresponding to the cphA1 fragment could be amplified by PCR from bacterial colonies recovered from surface-sterilized alfalfa nodules induced by the two recombinant strains Rm1021 (pBBR1MCS-3::cphA1 ${ }_{7120}$ ) and Rm4775 (pBBR1MCS$\left.3:: c p h A 1_{7120}\right)$. In addition, a 4,134-bp fragment corresponding to the cphB1-cphA1 fragment could be amplified by PCR from the bacterial colonies recovered from surface-sterilized alfalfa nodules induced by the two recombinant strains Rm1021 (pBBR1MCS-3::cphB1 $1:: c p h A 1_{7120}$ ) and Rm4775 (pBBR1MCS-3::cphB1 $1_{7120}:: c p h A 1_{7120}$ ), demonstrating the presence of these plasmids in at least some bacterial cells during symbiosis 35 or 55 days after inoculation. 


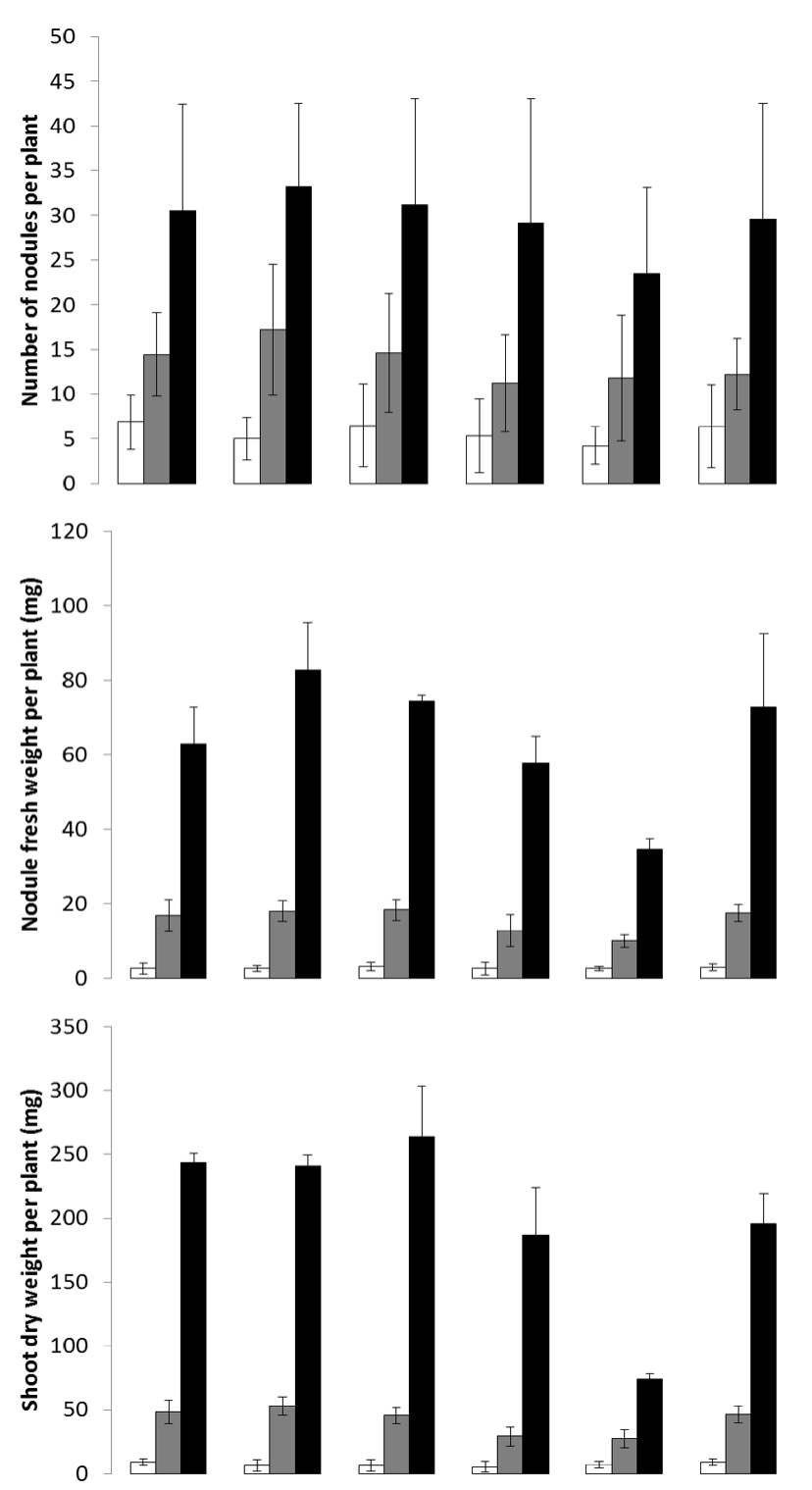

Figure 1: Time course of growth of alfalfa plants inoculated with different $S$. meliloti strains. Plants were inoculated with cells of (left to right) $S$. meliloti 1021 (wild type), S. meliloti 1021 (pBBR1MCS-3::cphA1 $1_{7120}$ ), S. meliloti 1021 (pBBR1MCS-3::cphB1 $\left.::: c p h A 1_{7120}\right)$, S. meliloti 1021 phbC $2 \mathrm{Km}$, S. meliloti 1021 phbC $\mathrm{Km}$ (pBBR1MCS-3::cphA1 ${ }_{7120}$ ), or S. meliloti 1021 phbC $\mathrm{Km}$ (pBBR1MCS-3::cphB1 $\left.1_{7120}:: c p h A 1_{7120}\right)$. Plants were grown in a growth chamber under the conditions described in the Materials and Methods section. The values of the parameters are means \pm standard deviation from 8 plants at 15 days post-inoculation (white bars), 10 plants at 35 days post-inoculation (grey bars), and 30 plants at 55 days post-inoculation (black bars).

\section{Ultrastructure of nodules}

Electron micrographs of sections of alfalfa nodules induced by the $\mathrm{PHB}^{+}$S. meliloti strains showed that PHB inclusions were present in the cytoplasm of the bacterial cells in the infection thread and also just after release of the bacterial cells into the host plant cells, but absent from the mature bacteroids.

Compact electron-dense bodies were observed within the cytoplasm of mature bacteroids in all nodules sections of all treatments as well as in the wild type bacteroids (Figure 4). Only two differences were observed: (i) The sizes of the electron-dense bodies within the bacteroids of the recombinant strains Rm1021 and Rm4775 harbouring cphA1 alone were greater and the bodies exhibited irregular shapes, presumably due to the accumulation of CGP (Figure 4). Similar irregular shapes of CGP granules were observed in the cytoplasm of the bacterial cells of the strains when cultivated in YMB medium [27] and also in the cytoplasm of the cells of Acinetobacter baylyi strain ADP1 when cultivated in MSM medium [37]; (ii) Higher levels of rough endoplasmic reticulum (RER) and Golgi membranes were observed in plant cells containing bacteroids of the recombinant strain Rm1021 (pBBR1MCS-3::cphB1 1 : $:: p p h A 1_{7120}$ ) in comparison to plant cells containing bacteroids of the wild type strain or bacteroids of the other S. meliloti strains (Figure 4).

\section{Discussion}

In this study, we addressed the question whether or not the capability of CGP biosynthesis alone or of a combination of biosynthesis and degradation of CGP improves the capacity of recombinant PHBpositive or PHB-negative S. meliloti 1021 strains to fix atmospheric nitrogen, and whether or not the inoculated alfalfa host plants exhibited enhanced growth.

One striking result was that inactivation of $p h b C$ in the genome of Rm1021 negatively affected symbiosis. The plants, which were inoculated with the $p h b C$-negative mutant strain ( $R m 4775)$, showed significantly lower shoot dry mass and shoot nitrogen content in comparison to plants inoculated with the wild type strain. This negative effect was also observed by Aneja et al. [38], who reported that alfalfa plants inoculated with a $p h b C$-negative mutant of $S$. meliloti showed a delayed appearance of nodules, a reduced total number of nodules, and also a reduced shoot dry mass in comparison to plants inoculated with the wild type strain.

Bacteroids occupying indeterminate nodules, such as S. meliloti, $M$. ciceri and R. leguminosarum bv. viciae, do not accumulate PHB during symbiosis, but possess large numbers of PHB granules during the initial stages of invasion and cell division within the infection thread [23]. These PHB granules disappear during differentiation, and mature

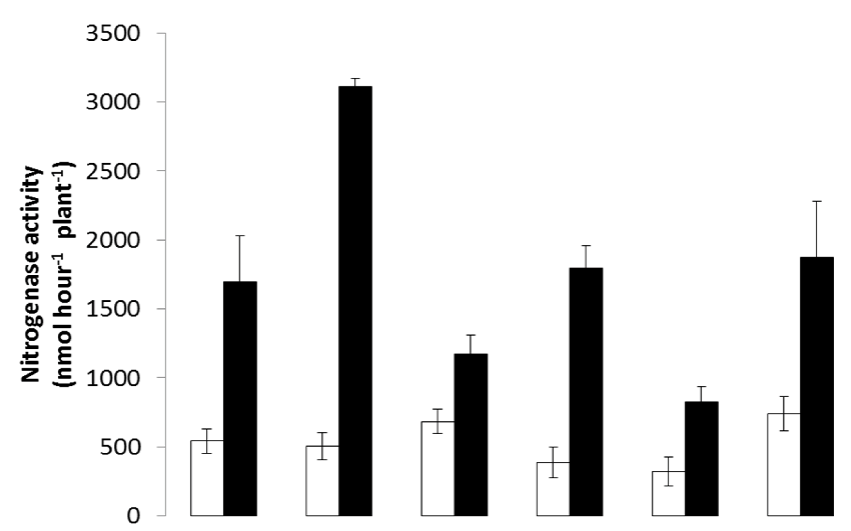

Figure 2: Nitrogenase activity of alfalfa plants inoculated with different $S$. meliloti strains. Plants were inoculated with cells of (left to right) $S$. meliloti 1021 (wild type), S. meliloti 1021 (pBBR1MCS-3::cphA1 ${ }_{7120}$ ), S. meliloti 1021 (pBBR1MCS-3::cphB1 $1_{7120}:: c p h A 1_{7120}$ ), S. meliloti 1021 phbC $2 \mathrm{Km}$, (e) S. meliloti 1021 phbC $2 \mathrm{Km}$ (pBBR1MCS-3::cphA1 ${ }_{7120}$ ), or S. meliloti 1021 phbC $\Omega \mathrm{Km}$ (pBBR1MCS-3::cphB1 $1_{7120}:: c p h A 1_{7120}$ ). Plants were grown in a growth chamber under the conditions described in the Materials and Methods section. The values are means \pm standard deviations from 10 plants at 35 days post-inoculation (white bars) and from 30 plants at 55 days postinoculation (black bars). 


\begin{tabular}{|c|c|c|c|c|c|c|c|c|}
\hline S.No & Shoot length $(\mathrm{cm})$ & Root length (cm) & Leaves/plant & Nodules/plant & $\begin{array}{l}\text { Nodule fresh } \\
\text { wt. (mg/plant) }\end{array}$ & $\begin{array}{l}\text { Shoot dry wt. } \\
\text { (mg/plant) }\end{array}$ & $\begin{array}{l}\% \mathrm{~N} \text { of shoot } \\
\text { dry wt. }\end{array}$ & $\begin{array}{l}\text { Nitrogenase } \\
\text { activity }\end{array}$ \\
\hline 1 & $55.68 \pm 4.98^{A}$ & $16.53 \pm 0.62^{\mathrm{a}}$ & $92.43 \pm 10.21^{\mathrm{AB}}$ & $30.30 \pm 2.06^{b}$ & $71.26 \pm 6.44^{B}$ & $289.35 \pm 1.60^{A}$ & $6.10 \pm 0.21^{\mathrm{A}}$ & $1675 \pm 164^{\mathrm{B}}$ \\
\hline 2 & $58.82 \pm 4.21^{\mathrm{A}}$ & $16.38 \pm 0.68^{a}$ & $96.79 \pm 8.69^{A}$ & $32.77 \pm 1.60^{b}$ & $94.47 \pm 7.76^{A}$ & $289.57 \pm 2.73^{A}$ & $4.90 \pm 0.31^{\mathrm{B}}$ & $3093 \pm 10^{A}$ \\
\hline 3 & $59.33 \pm 4.90^{A}$ & $14.77 \pm 0.57^{a}$ & $93.07 \pm 8.34^{\mathrm{AB}}$ & $31.40 \pm 2.08^{b}$ & $85.09 \pm 1.10 \mathrm{AB}$ & $317.68 \pm 23.69^{A}$ & $6.70 \pm 0.23^{A}$ & $1144 \pm 70^{c}$ \\
\hline 4 & $47.25 \pm 4.02^{\mathrm{A}}$ & $16.55 \pm 0.82^{\mathrm{a}}$ & $69.5 \pm 6.80^{\mathrm{B}}$ & $28.70 \pm 2.46^{b}$ & $66.45 \pm 4.33^{B}$ & $218.49 \pm 24.67^{B}$ & $4.77 \pm 0.26^{\mathrm{B}}$ & $1777 \pm 77^{\mathrm{B}}$ \\
\hline 5 & $24.13 \pm 3.05^{B}$ & $17.77 \pm 0.85^{\mathrm{a}}$ & $41.7 \pm 5.99^{c}$ & $22.53 \pm 1.90^{\mathrm{a}}$ & $39.85 \pm 1.59^{c}$ & $84.78 \pm 1.97^{c}$ & $4.10 \pm 0.23^{B}$ & $800 \pm 50^{c}$ \\
\hline 6 & $51.33 \pm 4.82^{A}$ & $16.93 \pm 0.59^{a}$ & $76.77 \pm 9.09^{\mathrm{AB}}$ & $28.57 \pm 2.26^{b}$ & $82.99 \pm 12.54^{\mathrm{AB}}$ & $235.62 \pm 17.60^{B}$ & $4.83 \pm 0.20^{B}$ & $1854 \pm 223^{B}$ \\
\hline
\end{tabular}

Table 2: Grow th parameters of alfalfa plants 55 days after inoculation $w$ ith different $S$. meliloti strains. Identical (a, b, c or A, B, C) superscripts $w$ ithin the same column denote values not significantly different at tw o significance levels: $P<0.05$ for $a, b$ and $c ; P<0.01$ for $A, B$ and $C$ ). The values of all parameters are mean values

\pm standard errors as obtained from the analyses of 30 plants inoculated $w$ ith the respective strains. Plants $w$ ere

inoculated w ith strains (1) S. meliloti 1021 (w ild type); (2) S. meliloti 1021 (pBBR1MCS-3::cphA17120); (3) S. meliloti 1021 (pBBR1MCS-3::cphB17120::cphA17120); (4)

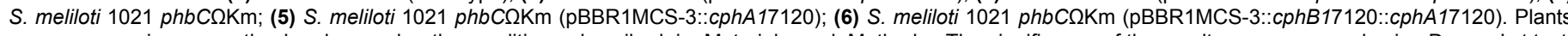
$\mathrm{w}$ ere grow $\mathrm{n}$ in a grow th chamber under the conditions described in Materials and Methods. The significance of the results $w$ as assessed using Duncan's test. Nitrogenase activity is reported as nmol of ethylene produced per hour per plant.
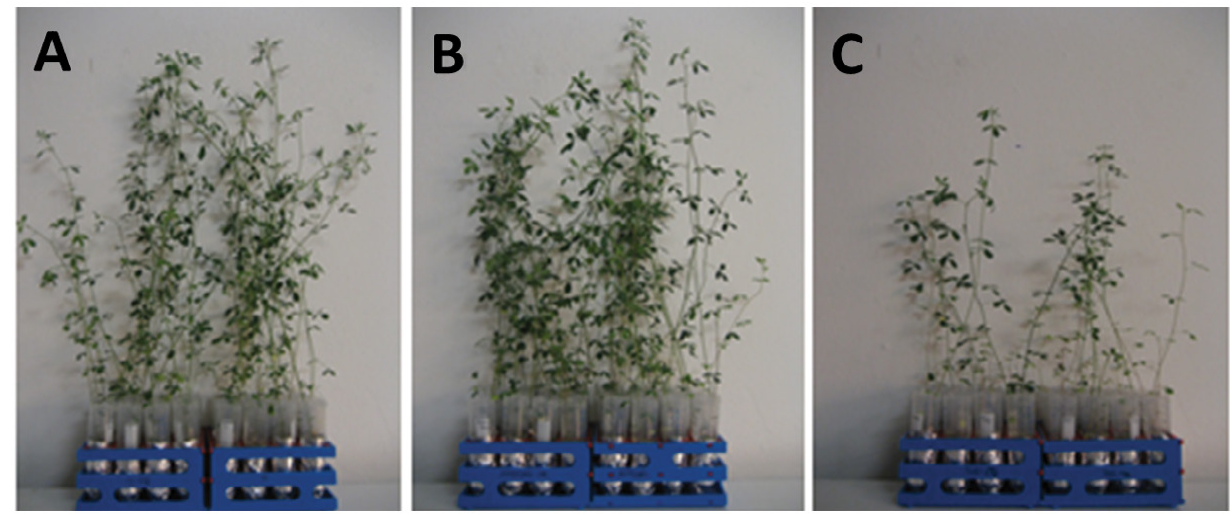

Figure 3: Images of 55-day old alfalfa plants after inoculation with (A) S. meliloti 1021 wild type, (B) S. meliloti 1021 (pBBR1MCS-3::cphB1 $1_{7120}:: c p h A 1_{7120}$ ), or (C) S. meliloti 1021 phbC $\Omega \mathrm{Km}$ (pBBR1MCS-3::cphA1 ${ }_{7120}$ ). Plants were grown in a growth chamber under the conditions described in the Materials and Methods section.

bacteroids are notably devoid of visible PHB granules [39]. The reason for this is not known; however, it has been shown that the activities of $\beta$-ketothiolase, acetoacetyl-CoA reductase, PHB depolymerase, and $\beta$-hydroxybutyrate dehydrogenases are similar in free-living cells and bacteroids of $M$. ciceri CC1192. Because gas chromatographic analysis also revealed only small amounts of PHB in bacteroids, PHB synthesis is presumably accompanied by an equivalent rate of degradation [29]. Although PHB mutants are capable of nodulation and of establishing effective symbiosis [40,41], it is possible that intracellular PHB may fuel cell division and growth during root infection and invasion [28].

The major exchange of nutrients between the symbiotic partners is the transfer of reduced carbon from the plant to support the nitrogenase activity of the bacteroids and the transfer of fixed nitrogen as ammonium ions from the bacteroids [25] in the opposite direction. The transferred ammonia is assimilated as glutamine in the plant cytoplasm by glutamine synthetase, and is then transferred to form other amino acids $[24,28,42]$. ATP, which is required for the reduction of nitrogen to ammonia, is derived from the reduced carbon provided by the plant $[24,43]$. CGP biosynthesis was also identified as an energyconsuming process [21]. As a result, CGP biosynthesis may compete with nitrogen fixation for ATP produced by the metabolism of C4dicarboxylates as sole energy source via the TCA cycle.

CGP biosynthesis in bacteroids probably has complex effects on nitrogen fixation due to the consumption of ATP and of amino acids. The consequences are lower plant growth, lower plant yields, and lower plant nitrogen content. The legume-rhizobial symbiosis responds to inefficient nitrogen fixation in complex ways, such as increased nodule mass as well as increased nodule number. An increase in nodule mass similar to what may be observed in inefficient symbioses is observed in the symbiosis induced by the recombinant strain Rm1021 (pBBR1MCS-3::cphA1 $1_{7120}$ ), although this symbiosis also exhibited a very high nitrogen fixation activity. However, this higher nitrogenase activity is not accompanied by higher rates of nitrogen assimilation by the plant, and the nitrogen contents of these plants are significantly reduced at 55 days. CGP is a nitrogen-rich polymer, and at least a portion of the fixed nitrogen may become locked up in CGP instead of being available to the plant. Similarly, production of CGP in transgenic tobacco and potato plants decreased the growth rate by the depletion of the amino acid pools of the plant [25]. A reduced ability to produce essential proteins might occur due to depletion of the amino acids, most likely of arginine and/or aspartate, caused by CGP production and storage [17]. Similarly, cell densities of transgenic Saccharomyces cerevisiae expressing $c p h A_{6308}$ reached lower values than those of the negative controls without $\operatorname{cph} A$, indicating that synthesis and accumulation of CGP resulted in slower growth [20].

Generally, simultaneous biosynthesis and degradation of CGP in bacteroids slightly improved the growth of the plants inoculated with the respective strains, although this increase could not be shown to be statistically significant. The slightly enhanced growth of the plants infected with these strain may be explained by additional 


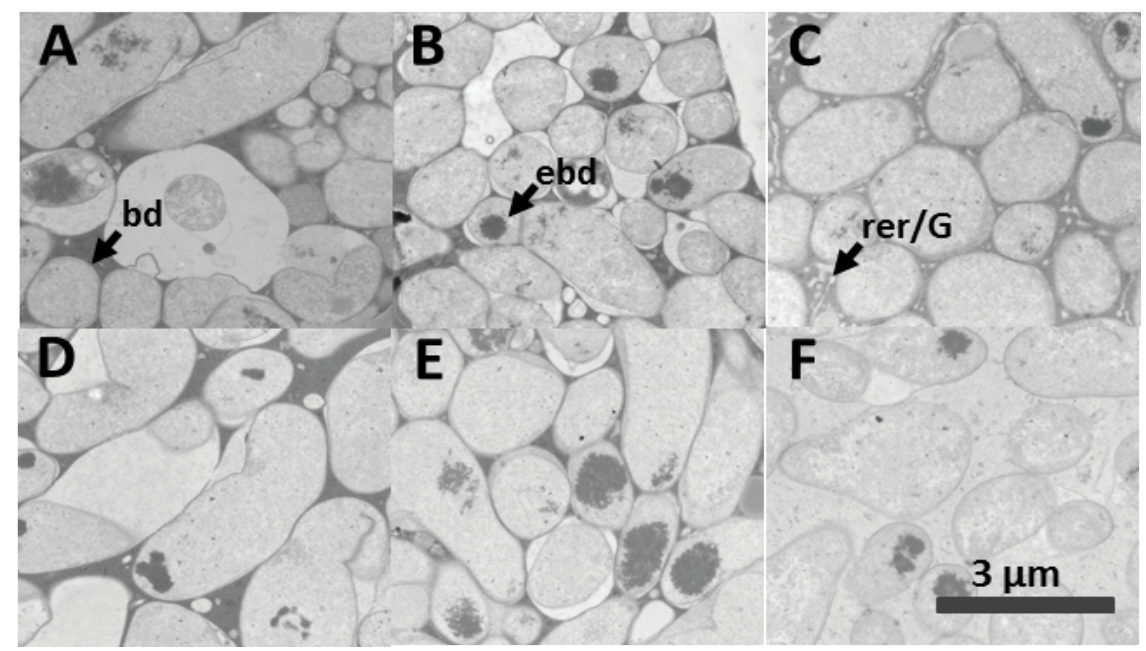

Figure 4: TEM photographs of thin sections of alfalfa nodules at 35 days post-inoculation with various $S$. meliloti strains. (A) Wild type strain $S$. meliloti 1021 , (B) S.

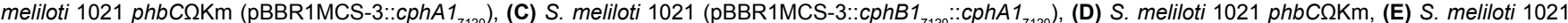

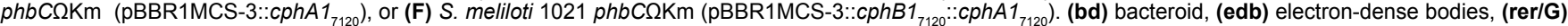
rough endoplasmic reticulum and/or Golgi bodies.

aspartic acid and arginine being released from CGP degradation via $\beta$-aspartate-arginine dipeptides produced by $\mathrm{CphB}$ and a peptidase of the bacteroids. The existence of the latter enzyme, which is responsible for cleavage of the dipeptides into the amino acid monomers, was confirmed by in vitro experiments [27]. At least in case of the $\mathrm{PHB}^{+}$ strain, similar or even enhanced growth parameters compared to the wild type were achieved at significantly reduced levels of nitrogen fixation. This observation may also suggest a more efficient transfer of nitrogen to the plant via CGP degradation products in these symbioses, and should be investigated more closely.

The compact electron-dense bodies that were observed within the cytoplasm of mature bacteroids of all S. meliloti strains probably consisted of polyphosphate [44]. Larger electron-dense bodies than those seen in the nodules induced by the wild type strain occurred in all nodules induced by the recombinant strains harbouring only $c p h A 1$ and are likely to be CGP granules. Further examination should be done to differentiate between the various electron-dense bodies within the bacteroids.

The large expansion of rough endoplasmic reticulum (RER) and Golgi bodies observed in the nodules induced by the recombinant strain Rm1021 (pBBR1MCS-3::cphB1 $17120:: \operatorname{cph} A 1_{7120}$ ) may be indicative of a high metabolic activity, which may be caused by enhanced ammonia levels from assimilation of the amino acids released from CGP degradation. The Golgi apparatus is involved in processing a wide range of cellular constituents, such as phosphatidylcholine (a component of the peribacteroid membrane structure), which is made in the endoplasmic reticulum and distributed via the Golgi membranes [45]. In addition, expanded rough endoplasmic reticulum (RER) and Golgi membranes were also seen in younger tissues [bacteroids not fully elongated] of effective nodules induced by $S$. meliloti wild type as well as in ineffective nodules induced by nitrogenase-defective $S$. meliloti Tn5 mutants [39]. These elevated levels may reflect synthesis and export of nodulins, which are normally repressed in effective nodules by the synthesis of nitrogenase or the production of ammonia [39]. Similar increases in rough endoplasmic reticulum (RER) extension and dilation, together with the formation of vesicles and vacuoles originating from the RER and Golgi, were observed in pea and soybean root nodules after host cell infection $[46,47]$. It was suggested that endocytosed rhizobia come into contact with host cell debris and RER- and Golgi-derived substances and that some of these substances might serve as substrates for rhizobial metabolism and differentiation.

Although the increases in growth observed in plants infected with strains to which an artificial CGP metabolism had been introduced were not statistically significant, the differences were consistently observed, and the system has not yet been optimized in any way. An increase of some growth parameters by about $10 \%$ is therefore remarkable. This study will pave the way to further enhance the growth and to increase the yield of legume crops.

\section{Acknowledgement}

We thank Ursula Malkus (Institut für Medizinische Physik und Biophysik) for expert preparation of the electron microscopic specimens and Katja Kemper (Institut für Molekulare Mikrobiologie und Biotechnologie) for her help in the harvest of the plants. We are grateful to Dr. Hanan Hassan (Faculty of Agriculture, Fayoum University, Egypt) for her help in the statistical analysis of the data. This study was supported by a fellowship provided by the Faculty of Agriculture, Cairo University, Egypt to Yasser Abd-El-Karem.

\section{References}

1. Borzi A (1887) Le comunicazioni intracellulari delle Nostochinee. Malpighia 1 : 28-74.

2. Simon RD, Weathers $P$ (1976) Determination of the structure of the novel polypeptide containing aspartic acid and arginine which is found in cyanobacteria. Biochim Biophys Acta 420: 165-176.

3. Allen MM (1988) Inclusions: Cyanophycin. Methods Enzymol 167: 207-213.

4. Simon RD (1987) Inclusion bodies in the cyanobacteria: Cyanophycin polyphosphate, polyhedral bodies. The cyanobacteria, Elsevier, Amsterdam, The Netherlands.

5. Wingard LL, Miller SR, Sellker JM, Stenn E, Allen MM, et al. (2002) Cyanophycin production in phycoerythrin-containing marine Synechococcus strain of unusual phylogenetic affinity. Appl Environ Microbiol 68: 1772-1777.

6. Krehenbrink M, Oppermann-Sanio FB, Steinbüchel A (2002) Evaluation of noncyanobacterial genome sequences for occurrence of genes encoding proteins homologous to cyanophycin synthetase and cloning of active cyanophycin synthetase from Acinetobacter sp. strain DSM 587. Arch Microbiol 177: 371-380.

7. Ziegler K, Deutzmann R, Lockau W (2002) Cyanophycin synthetase-like enzymes of non-cyanobacterial eubacteria: Characterization of the polymer produced by a recombinant synthetase of Desulfitobacterium hafniense. Z Naturforsch C 57: 522-529. 
Citation: Abd-El-Karem Y, Reichelt R, Krehenbrink M, Steinbüchel A (2016) Effect of Cyanophycin Metabolism in Recombinant Sinorhizobium (Ensifer) meliloti 1021 on the Symbiosis with Alfalfa (Medicago sativa). J Microb Biochem Technol 8: 375-381. doi: 10.4172/1948-5948.1000312

8. Lang NJ Simon RD, Wolk CP (1972) Correspondence of cyanophycin granules with structured granules in Anabaena cylindrica. Arch Microbiol 83: 313-320.

9. Gupta M, Carr NG (1981) Enzyme activities related to cyanophycin metabolism in heterocysts and vegetative cells of Anabaena spp. J Gen Microbiol 125 17-23.

10. Hejazi M, Piotukh K, Mattow J, Deutzmann R, Volkmer-Engert R, et al. (2002) Isoaspartyl dipeptidase activity of plant-type asparaginases. Biochem $\mathrm{J} 364$ 129-136.

11. Füser G, Steinbüchel A (2007) Analysis of genome sequences for genes of cyanophycin metabolism: identifying putative cyanophycin metabolizing prokaryotes. Macromol Biosci 7: 278-296.

12. Picossi S, Valladares A, Flores E, Herrero A (2004) Nitrogen-regulated genes for the metabolism of cyanophycin, a bacterial nitrogen reserve polymer: Expression and mutational analysis of two cyanophycin synthetase and cyanophycinase gene clusters in the heterocyst-forming cyanobacterium Anabaena sp. PCC7120. J Biol Chem 279: 11582-11592.

13. Aboulmagd E, Oppermann-Sanio FB, Steinbüchel A (2000) Molecular characterization of the cyanophycin synthetase from Synechocystis sp. strain PCC6308. Arch Microbiol 174: 297-306.

14. Berg H, Ziegler K, Piotukh K, Baier K, Lockau, et al. (2000) Biosynthesis of the cyanobacterial reserve polymer multi-L-arginyl-poly-L-aspartic acid (cyanophycin) Mechanism of the cyanophycin synthetase reaction studied with synthetic primers. Eur J Biochem 267: 5561-5570.

15. Hai T, Oppermann-Sanio FB, Steinbüchel A (1999) Purification and characterization of cyanophycin and cyanophycin synthetase from the thermophilic Synechococcus sp. MA19. FEMS Microbiol Lett 181: 229-236.

16. Aboulmagd E, Voss I, Oppermann-Sanio FB, Steinbüchel A (2001) Heterologous expression of cyanophycin synthetase and cyanophycin synthesis in the industrial relevant bacteria Corynebacterium glutamicum and Ralstonia eutropha and in Pseudomonas putida. Biomacromolecules 2: 13381342.

17. Hühns M, Neumann K, Hausmann T, Klemke F, Lockau W, et al. (2009) Tuberspecific cphA expression to enhance cyanophycin production in potatoes. Plan Biotechnol J 7: 883-898.

18. Neumann K, Stephan DP, Ziegler K, Hühns M, Broer I, et al. (2005) Production of cyanophycin, a suitable source for the biodegradable polymer polyaspartate, in transgenic plants. Plant Biotechnol J 3: 249-258.

19. Steinle A, Bergander K, Steinbüchel A (2009) Metabolic engineering of Saccharomyces cerevisiae for production of novel cyanophycins with an extended range of constituent amino acids. Appl Environ Microbiol 75: 3437 3446 .

20. Steinle A, Oppermann-Sanio FB, Reichelt R, Steinbüchel A (2008) Synthesis and accumulation of cyanophycin in transgenic strains of Saccharomyces cerevisiae. Appl Environ Microbiol 74: 3410-3418.

21. Steinle A, Witthoff S, Krause JP, Steinbüchel A (2010) Establishment of cyanophycin biosynthesis in Pichia pastoris and optimization by use of engineered cyanophycin synthetases. Appl Environ Microbiol 76: 1062-1070.

22. van Rhijn P, Vanderleyden J (1995) The Rhizobium-plant symbiosis. Microbiol Rev 59: 124-142.

23. Gage DJ, Bobo T, Long SR (1996) Use of green fluorescent protein to visualize the early events of symbiosis between Rhizobium meliloti and alfalfa (Medicago sativa). J Bacteriol 178: 7159-7166.

24. Udvardi MK, Day DA (1997) Metabolite transport across symbiotic membranes of legume nodules. Annu Rev Plant Physiol Plant Mol Biol 48: 493-523.

25. Bergersen JF, Turner GL (1967) Nitrogen fixation by the bacteroid fraction of breis of soybean root nodules. Biochim Biophys Acta 141: 507-515.

26. Lodwig EM, Hosie AH, Bourdès A, Findlay K, Allaway D, et al. (2003) Aminoacid cycling drives nitrogen fixation in the legume-Rhizobium symbiosis. Nature 422: $722-726$.

27. Abd-El-Karem Y, Elbers T, Reichelt R, Steinbüchel A (2011) Heterologous expression of Anabaena sp. PCC7120 cyanophycin metabolism genes cphA1 and cphB1 in Sinorhizobium (Ensifer) meliloti 1021. Appl Microbiol Biotechnol 89: 1177-1192.

28. Lodwig E, Poole P (2003) Metabolism of Rhizobium bacteroids. Crit Rev Plant Sci 22: 37-78.
29. Kim SA, Copeland L (1996) Enzymes of Poly-(beta)-hydroxybutyrate metabolism in soybean and chickpea bacteroids. Appl Environ Microbiol 62 4186-4190.

30. Somasegaran P, Hoben HJ (1994) Handbook for Rhizobia. Methods in Legume-Rhizobium Technology, Springer Laboratory, New York.

31. Miller-Williams M, Loewen PC, Oresnik IJ (2006) Isolation of salt-sensitive mutants of Sinorhizobium meliloti strain Rm1021. Microbiology 152: 2049 2059

32. Broughton WJ, Dilworth MJ (1971) Control of leghaemoglobin synthesis in snake beans. Biochem J 125: 1075-1080.

33. Hafez AR, Mikkelsen DS (1981) Colorimetric determination of nitrogen for evaluation the nutritional status of rice. Commun Soil Sci Plant Anal 12: 61-69.

34. Arnold M (1968) Histochemie. Einführung in die Grundlagen und Prinzipien der Methoden. Springer, Berlin, Germany.

35. Spurr AR (1969) A low-viscosity epoxy resin embedding medium for electron microscopy. J Ultrastruct Res 26: 31-43.

36. Reynolds ES (1963) The use of lead citrate at high $\mathrm{pH}$ as an electron-opaque stain in electron microscopy. J Cell Biol 17: 208-212.

37. Elbahloul Y, Krehenbrink M, Reichelt R, Steinbüchel A (2005) Physiological conditions conducive to high cyanophycin content in biomass of Acinetobacter calcoaceticus strain ADP1. Appl Environ Microbiol 71: 858-866.

38. Aneja P, Zachertowska A, Charles TC (2005) Comparison of the symbiotic and competition phenotypes of Sinorhizobium meliloti PHB synthesis and degradation pathway mutants. Can J Microbiol 51: 599-604.

39. Hirsch AM, Bang M, Ausubel FM (1983) Ultrastructural analysis of ineffective alfalfa nodules formed by nif::Tn5 mutants of Rhizobium meliloti. J Bacterio 155: $367-380$.

40. Povolo S, Tombolini R, Morea A, Anderson AJ, Casella S, et al. (1994) Isolation and characterization of mutants of Rhizobium meliloti unable to synthesize poly-ß-hydroxybutyrate (PHB). Can J Microbiol 40: 823-829.

41. Willis LB, Walker GC (1998) The phbC (poly-beta-hydroxybutyrate synthase) gene of Rhizobium (Sinorhizobium) meliloti and characterization of phbC mutants. Can J Microbiol 44: 554-564.

42. Cullimore JV, Bennett MJ (1988) The molecular biology and biochemistry of plant glutamine synthetase from root nodules of Phaseolus vulgaris L. and other legumes. J Plant Physiol 132: 387-393.

43. Schrauzer GN (1975) Non-enzymatic simulation of nitrogenase reactions and the mechanism of biological nitrogen fixation. Angew Chem Int Ed Engl 14 514-522.

44. Craig AS, Williamson KI (1972) Three inclusions of rhizobial bacteroids and their chemical character. Arch Microbiol 87: 165-171.

45. Robertson JG, Lyttleton P, Bullivant S, Grayston GF (1978) Membranes in lupin root nodules. I. The role of Golgi bodies in the biogenesis of infection threads and peribacteroid membranes. J Cell Sci 30: 129-149.

46. Kijne JW, Planque K (1979) Ultrastructural study of the endomembrane system in infected cells of pea and soybean root nodules. Physiol Plant Pathol 14 339-345.

47. Meade HM, Long SR, Ruvkun GB, Brown SE, Ausubel FM (1982) Physical and genetic characterization of symbiotic and auxotrophic mutants of Rhizobium meliloti induced by transposon Tn5 mutagenesis. J Bacteriol 149: 114-122. 